\section{GRP-051 DISPENSING PRACTISE IN SAUDI COMMUNITY PHARMACY}

doi:10.1136/ejhpharm-2013-000276.051

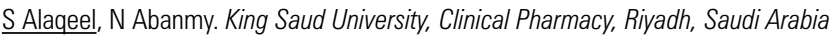

Background Community pharmacies are the first point of contact in the healthcare system. Thus, community pharmacists have a crucial role in optimising the medicines use process and promoting patient outcomes.

Purpose The study aimed to examine counselling and dispensing practise in community pharmacy in Riyadh city, the capital of Saudi Arabia.

Materials and Methods The simulated patient (SP) method was used to measure how pharmacists provided patient counselling. There were four scenarios. Scenarios 1 and 2 concerned drug-drug and drug-food interactions, scenario 3 concerned the proper time of administration, and scenario 4 concerned side effects. Each pharmacy was visited twice with different scenarios. The simulated visits were conducted between April and May, 2012. A questionnaire to test the attitude of community pharmacists regarding counselling and dispensing practise was distributed in the same period.

Results There were 161 simulated visits. Community pharmacists did not ask SPs any questions during 144 (90\%) visits. Pharmacists did not provide any information to SPs during 152 (95\%) visits. When the SPs asked specific questions about their medicines, pharmacists provided no information during 30 (19\%) visits. 350 questionnaires were distributed. Of the respondents, 232 (64\%) reported that they usually or always tell the patient about the purpose of medicines or the diagnosis, $302(98 \%)$ reported that they usually or always give patient information on how to use or apply the medicine. 299 (85\%) said they were satisfied with their counselling practise.

Conclusions Dispensing practise in the community in Saudi Arabia seems inadequate. There is a strong need to improve medicines counselling and dispensing practise in community pharmacies.

No conflict of interest.

\section{GRP-052 DOES AN INCREASE IN THE USE OF LOW MOLECULAR WEIGHT HEPARIN THROMBOPROPHYLAXIS CORRESPOND TO A DECREASE IN THE INCIDENCE OF HOSPITAL- ACOUIRED VENOUS THROMBOEMBOLISM?}

doi:10.1136/ejhpharm-2013-000276.052

L Hammond, F Kennedy, N O'Hanlon, J O'Shea. St. Vincent's University Hospital, Pharmacy Department, Dublin, Ireland (Rep.)

Background Venous thromboembolism (VTE) is a common complication of hospital admission. Pulmonary Embolism (PE) accounts for $5-10 \%$ of deaths in hospitalised patients and is the most common cause of preventable hospital mortality. Prophylactic LowMolecular-Weight heparin (LMWH) reduces the risk of VTE but is widely under-prescribed. Although LMWH prophylaxis in SVUH has been cyclically audited since 2007, the use of thromboprophylactic LMWH has not been studied in comparison to the incidence of hospital-acquired VTE.

Purpose To measure the use of LMWH thromboprophylaxis and to compare this to the rate of confirmed hospital-acquired VTE.

Materials and Methods The pharmacy dispensing and stock management system provided data on the use of thromboprophylactic LMWH from 2007 onwards. Data on the incidence of hospitalacquired VTE was collected from the Hospital Information System. These figures were compared with one another.

Results The rate of use of thromboprophylactic LMWH in SVUH rose by $26 \%$ over the study period. The average incidence of hospital-acquired VTE was 8.3 (range 6.8-9.3) per 1,000 inpatient admissions over the same period. This average is consistent with published rates, but the incidence in SVUH increased over the study period.

Conclusions Hospital-acquired VTE is a major public health issue and is associated with substantial morbidity and mortality. Appropriate thromboprophylaxis is considered to be the most effective strategy for preventing VTE. Although the use of LMWH thromboprophylaxis in SVUH increased steadily over the study period, the incidence of VTE also increased over the same period, suggesting that there are other factors (e.g. patient complexity, inappropriate LMWH dosing etc.) influencing the rate of hospital-acquired VTE. Audit of LMWH thromboprophylaxis is a useful tool to assess awareness and compliance with in-hospital VTE prophylaxis guidelines. Trends in the incidence of hospital-acquired VTE may be helpful in assessing the effectiveness of in-hospital thromboprophylaxis, when other factors are taken into consideration.

No conflict of interest.

\section{GRP-053 DRUG DISTRIBUTION SYSTEMS: EFFECT ON MEDICATION ERROR RATES AND COST OF SAFETY IN A GERIATRIC SHORT STAY UNIT}

doi:10.1136/ejhpharm-2013-000276.053

'E Cousein, ' $\mathrm{J}$ Mareville, ${ }^{1} \mathrm{~A}$ Pruvost, ${ }^{2} \mathrm{C}$ Jost, ${ }^{2} \mathrm{G}$ Gommez, ${ }^{2} \mathrm{D}$ Dambre, ${ }^{1} \mathrm{P}$ Coupe 'Valenciennes' Hospital, Pharmacy Department, Valenciennes, France; 'Valenciennes' Hospital, Geriatric Short Stay Unit, Valenciennes, France

Background Medication management of older peoples is of special importance regarding to their sensitivity to adverse drugs effects.

Purpose This study compared the medication error rates and the operating costs before and after implementation of an automated unit dose distribution system:

- before: ward stock system (WSS): prescriptions in paper record, medications prepared by nurses in the ward,

- after: computerised physician order entry including electronic medication administration record (eMAR), pharmacist interventions, unit dose delivering system robot, automated medications delivering systems.

Materials and Methods Medication errors were identified using an observation-based method. Pharmacist attended drugs administration rounds in a randomly selected ward section. Administrations were compared to prescriptions. Error rates and error types were compared according to a chi square method. Clinical severity of errors was assessed.

Drug consumptions, costs of pharmaceutical and nursing staffs and equipment were calculated for each period.

Results During the WSS period, 28 rounds were attended (148 patients, 615 drugs administrations) versus 31 rounds (166 patients, 783 drugs administrations) during the UDDS period. The rate of medication errors significantly decreased between the WSS period and the UDDS period (12.6\% vs $5.2 \%$ ). During the WSS period, a medication error occurred by $30.4 \%$ of the patients (45 patients) compared to $19.9 \%$ (32 patients) during the UDDS period ( $p<0.05)$. Most reduced errors during the UDDS period were wrong dose (16 vs 4 ) and wrong drug (19 vs 1 ).

Drugs consumptions decreased of $11,527 €$ a year $(11.5 \%)$ and cost of nurses time saved was assessed at $24,642 €$ a year. One dose delivered by robot cost $0.56 €, 0.41 €$ excluding robot loan. Safety brought by the automated unit dose distribution system cost $90.4 €$ for one bed a month, including, staff and equipment.

Conclusions Drug safety showed her necessity, this has an additional cost witch must be compared with consequences of medication errors and medicine-related illness.

No conflict of interest. 